\title{
A model for long-term environmental sound detection
}

\author{
Dick Botteldooren, Member, IEEE, and Bert De Coensel, Member, IEEE
}

\begin{abstract}
In recent years, knowledge on primary processing of sound by the human auditory system has tremendously increased. This paper exploits the opportunities this creates for assessing the impact of (unwanted) environmental noise on quality of life of people. In particular the effect of auditory attention in a multisource context is focused on. The typical application envisaged here is characterized by very long term exposure (days) and multiple listeners (thousands) that need to be assessed. Therefore, the proposed model introduces many simplifications. The results obtained show that the approach is nevertheless capable of generating insight in the emergence of annoyance and the appraisal of open area soundscapes.
\end{abstract}

\section{INTRODUCTION}

The amount of experimental data on the functioning of the auditory system has been growing tremendously during the last couple of years, to a large extent due to the advent of several new brain imaging techniques. An interesting overview on the neurobiological basis of auditory attention, of interest for the work presented in this paper, can be found in [1]. This evolution stimulated the development of computer models for human auditory processing such as [2] or [3]. Most of these models focus on speech processing, which is obviously of utmost interest in many applications. However, auditory processing of environmental sound has rarely been assessed with the same amount of detail [4]. Much inspiration can be found in classical work on visual attention such as [5], but there are also significant differences caused by the different evolutionary use of the auditory and visual systems.

Classical environmental noise assessment basically focuses on reducing noise levels of unwanted sounds. Little or no attention was paid to detailed acoustical features of the sound, let alone to the meaning that the listener gave to this sound. More recently, the concept of (urban) soundscape design - an idea originating in the early seventies [6] has gained renewed interest [7], [8], [9], but virtually none of the soundscape researchers have used computer models for auditory perception in their approach. Nevertheless, it is at least conceptually - evident that an accurate computer model for auditory perception should be able to handle environmental sounds as well. In this paper, we address this issue in particular.

A first important difference between more common computer-modeling of auditory perception of speech and modeling the impact of environmental sounds on the everyday life of people is the totally different time scale involved. Processing speech is usually a matter of seconds

Dick Botteldooren and Bert De Coensel are with the Department of Information Technology (INTEC), Ghent University, Belgium (phone: +329-264-9968; fax: +32-9-264-9969; email: dick.botteldooren@intec.ugent.be, bert.decoensel@intec.ugent.be). or minutes. The appreciation of the soundscape of a natural green area may be based on the sounds heard over a period of a quarter of an hour. Evaluating the sonic quality of the living environment is a process that could take months. A second difference emerges from the very strong effect of personal factors on environmental noise perception. Amongst these personal factors are inter-individual psychophysical differences such as sensitivity to noise [10], [11], social context and lifestyle differences [12]. For these reasons, studies on the effect of environmental sound are always based on averages over vast numbers of people (typically several hundred to thousands). A computer model able to simulate these long exposures to environmental sounds for huge numbers of virtual people should be fast. This implies that a lot of simplifications are needed.

In Section II we present such a simplified model for hearing environmental sound and in Section III this model will be applied to several test cases.

\section{THE COMPUTER MODEL}

The core of the proposed computer model is the sound noticing and attention focusing module (Fig. 1). The input for this module can either be a set of simulated time series of environmental sounds or a measurement. The simulation considers individual vehicles (cars, trains, planes) [13] but treats natural ambient sounds as a whole rather than to model the sounds produced by each bird, each tree etc. For the latter, the loudness is assumed to fluctuate following a $1 / f$ characteristic typical for a complex system, since this characteristic was found in many recordings of environmental sounds [14]. In case recordings are used, the non-trivial problems of environmental sound recognition and auditory scene analysis (ASA) [15] have to be solved. We do not elaborate on this since the examples given in this paper will be based on simulations that automatically result in separate auditory streams for separate sound sources. The only ASA related problem that needs to be resolved is the detection of vehicle streams rather than individual vehicles if appropriate. To group events, a gating mechanism was introduced already in earlier computational work [16].

The output of the sound noticing and attention focusing module feeds into a module that covers cognitive processing. This extensive module attaches meaning to the sounds noticed, within a physical and expected context. The physical context encompasses mainly visual information, but also smell and feeling of temperature can contribute. Expectation is based on prior experience or knowledge transferred from other individuals verbally. Today, no computer models are available that can handle this part of the complex problem 


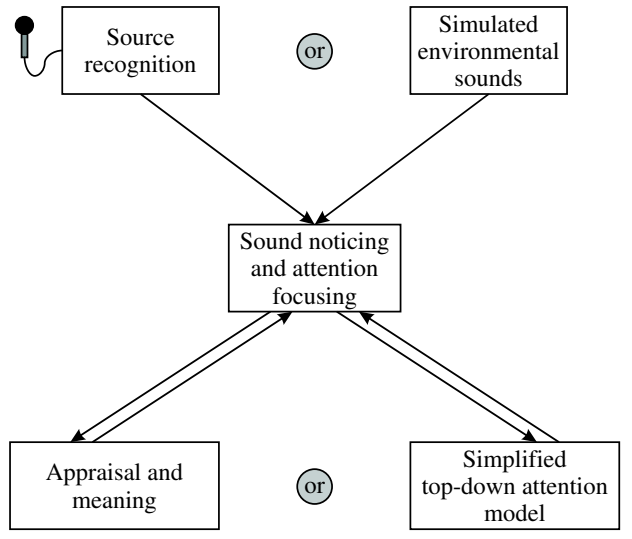

Fig. 1. Basic building blocks of the overall model.

but confabulation theory [17] or modeling field theory [18] are very good candidates.

It is widely acknowledged that attention focusing is directed using both bottom-up, sound-based cues and topdown, activity-dependent cues [1]; similar mechanisms have been identified in visual attention focusing [5], [19]. The central module in Fig. 1, which is in the primary scope of this paper, uses input from the higher level cognitive module via top-down attention focusing and by creating an expectation for the low level source identification. To provide this essential information, a very simplified model that provides the top-down attention for environmental sound in general is introduced:

$$
A_{\text {down }}=A_{e s, 0}+\alpha \int_{-\infty}^{t_{0}} \sum_{i} A_{e s, i}(t) \exp \left(\frac{t-t_{0}}{\tau_{e s}}\right) \mathrm{d} t
$$

where $A_{e s, 0}$ is the background attention for environmental sounds, which depends amongst others on the current activity and intentions of the modeled individual. For simplicity, this background attention is kept constant during simulation, implying that the modeled individual only takes part in a single activity. The second term in Eq. 1 expresses that bottom-up attention triggered by a particular environmental sound $\left(A_{e s, i}\right)$ may eventually increase top-down attention as well. One may refer to this as focusing. This term contains a factor $\alpha$ that weighs the importance of this effect and a time constant $\tau_{e s}$ that determines its adaptation speed.

In Fig. 2 the layout of the sound noticing and attention focusing module is shown. The environmental sounds are first put in the total auditory scene. This first processing step models the physiological limitations of the human ear and could account for physiological masking where all other sounds are regarded as a masking background for every particular sound in the set. A very rudimentary but fast estimate can be obtained by calculating the signal-to-noise ratio for every sound in the set.

A very important step in the process is the habituation and deviant or saliency detect. In human auditory processing, it is believed that a large part of this phenomenon is a pre-

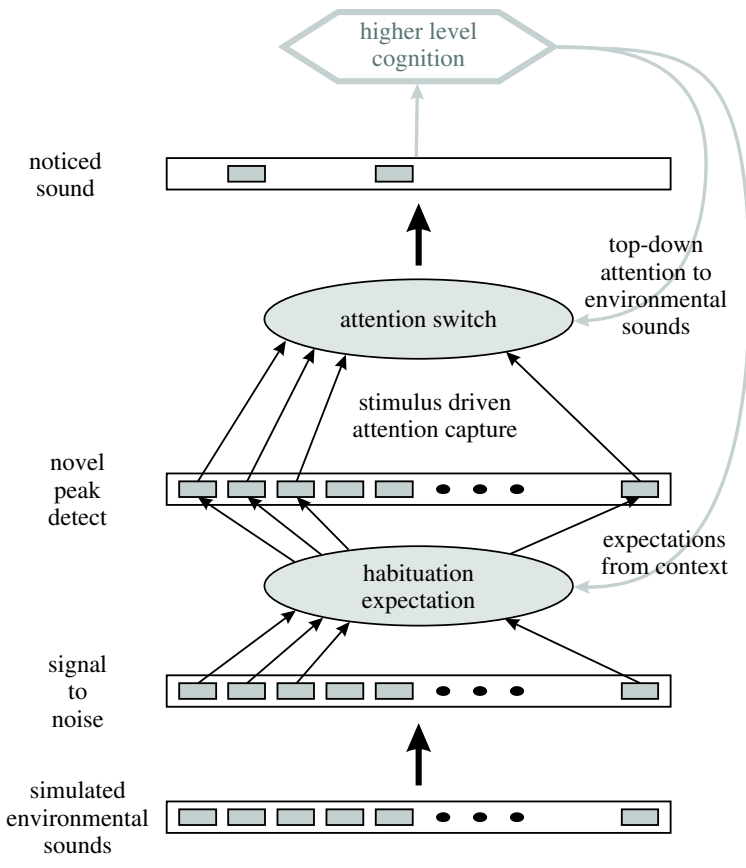

Fig. 2. Layout of the sound noticing and attention focusing module.

attentive process [1]. Hence it is included in the computer model also on the basis of primary sound signals. The simplest model for saliency in environmental sounds is a peak detection algorithm. Peaks are detected if they stand out of the average level. Since the peak detection is implemented for each individual type of sound in the complex acoustic environment, not much care is needed for deviant behavior on the frequency axis that normally is included in the calculation of a saliency map [20]. To include habituation in the peak detection, the signal-to-noise ratio for a particular source is reduced by an amount that depends linearly on the exponential average of past excitations $S(t)$ :

$$
C_{h, 0}+C_{h} \int_{-\infty}^{t_{0}} S(t) \exp \left(\frac{t-t_{0}}{\tau_{h}}\right) \mathrm{d} t
$$

where $\tau_{h}$ is the time constant for habituation.

When a peak detect occurs for a particular sound, it tries to attract attention. This bottom-up attention process is introduced in the model in a simple and fast way by an attention switch process that distributes available attention over sounds reaching the detection threshold. Rather than strictly limiting total attention, an amount of attention is focused on the newly detected sound that decreases as attention is already focused on another environmental sound,

$$
\Delta A_{u p}=\beta\left(A_{e s, d}-\sum_{i} A_{e s, i}(t)\right)
$$

where $A_{e s, d}$ is the dynamic part of attention and $\beta$ is a factor indicating which part of available attention is attributed to the newly detected sound. If the person-activity combination 
does not allow switching attention to environmental sounds easily, $A_{e s, d}$ should be kept small. If attention can not easily be switched between environmental sounds, $\beta$ should be kept small. The total level of attention attributed to the $i$ th environmental sound becomes:

$$
A_{\text {es }, i}(t)=A_{\text {down }}+\Delta A_{\text {up }} \exp \left(\frac{t_{i}-t}{\tau_{i}}\right)
$$

where $t_{i}$ is the instance of the last increase of attention and $\tau_{i}$ is a suitable time constant. This model implicitly includes attentional gating.

Attention and excitation level (signal-to-noise ratio) determine which environmental sounds are noticed. Note that the proposed model allows several environmental sounds to be noticed at the same time and thus share some of the listener's attention. This may seem surprising at first glimpse but is solely determined by the smallest time steps of the order of 1 second that are typically chosen for this type of simulation. During this time interval attention may actually switch up and down between both sources at hand, leading to a nonzero average level of attention for each.

To measure effects, three quantities are recorded: the number of times a sound is noticed, $N_{n}$, the time that the sound is noticed, $T_{n}$, and the above-threshold exposure level of the noticed sound, $S E L_{t h r}$. The latter is defined as the integrated strength of the sounds noticed, where strength is defined as the signal-to-noise ratio suitably modified to account for habituation (Eq. 2) and attention (Eq. 4).

\section{Simulation RESUlts}

The computer model for long-term environmental sound detection is applied to two case studies. In line with the usual epidemiological way of studying the overall effect of environmental sounds on people, several thousands of virtual individuals in as many sonic environments are simulated. Simulated results such as the time that a sound was heard are further analyzed statistically exactly as one would analyze results of field studies with biological agents. This eventually leads to trends that could be compared to literature data and field observations.

\section{A. Open area soundscape perception}

The first case study concerns the perception of open area soundscapes. The user of this area is assumed to be actively looking for recreation and psychological restoration. It can safely be assumed that this user is attentive for the natural environment, including its soundscape. Hence attention for environmental sounds is relatively high.

A typical but quite general situation for the open area soundscape that will be used in this case study is shown in Fig. 3. The listener is surrounded by natural sound and confronted with sounds from a highway, a road and a railway, located at respective distances $d_{h w}, d_{r d}$, and $d_{r w}$. In all, 10000 simulated situations underlie the results presented in the following graphs. The range of data describing the rural exposure to road and rail noise are given in Table I. These values can be regarded as typical for the open area in

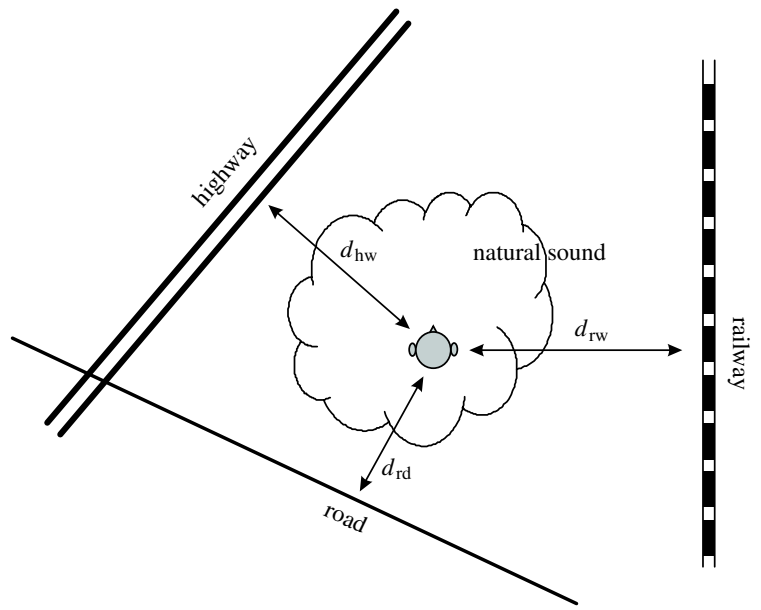

Fig. 3. Prototype situations for the open area that are studied.

TABLE I

RANGE OF DISTANCES AND TRAFFIC INTENSITIES USED IN THE NUMERICAL EXPERIMENT.

\begin{tabular}{llll}
\hline \hline Quantity & Average & Minimum & Maximum \\
\hline$d_{h w}$ & $3.9 \mathrm{~km}$ & $1 \mathrm{~km}$ & $10 \mathrm{~km}$ \\
$N_{h w}$ & 2000 cars $/ \mathrm{h}$ & 1500 cars $/ \mathrm{h}$ & 2500 cars $/ \mathrm{h}$ \\
& 400 trucks $/ \mathrm{h}$ & 300 trucks $/ \mathrm{h}$ & 500 trucks $/ \mathrm{h}$ \\
$d_{r d}$ & $185 \mathrm{~m}$ & $5 \mathrm{~m}$ & $1 \mathrm{~km}$ \\
$N_{r d}$ & 250 cars $/ \mathrm{h}$ & $5 \mathrm{cars} / \mathrm{h}$ & 500 cars $/ \mathrm{h}$ \\
& 10 trucks $/ \mathrm{h}$ & 1 truck $/ \mathrm{h}$ & 20 trucks $/ \mathrm{h}$ \\
$d_{r w}$ & $1950 \mathrm{~m}$ & $500 \mathrm{~m}$ & $5 \mathrm{~km}$ \\
$N_{r w}$ & 1 train $/ \mathrm{h}$ & No trains & 3 trains $/ \mathrm{h}$ \\
\hline \hline
\end{tabular}

northwest Europe, except for the number of trains which is rather low. A typical noise emission for the European fleet is used. Natural sound levels also differ between situations, with average levels ranging between 20 and $60 \mathrm{~dB}(\mathrm{~A})$ with a Gaussian distribution with spread of $12 \mathrm{~dB}(\mathrm{~A})$ and a $1 / f$ temporal structure.

Of particular interest for this paper is the effect that model parameters linked to auditory perception have on the trends observed in the simulations. The time that the sound is noticed, $T_{n}$, is used to visualize some of the effects. The data are further analyzed by average level of exposure to road traffic sound and average level of exposure to natural ambient sound.

Fig. 4 and Fig. 5 show $T_{n}$ as a function of ambient natural sound for different average levels of road traffic sound, for high and low top-down attention for environmental noise, respectively. The overall trend of these results corresponds to what could be expected. As ambient noise level grows, traffic noise is less noticeable. The effect is less for higher traffic noise levels. Similarly, natural sound is more often noticed as natural ambient sound levels grow and as traffic noise levels are less. Since modeled attention can not be judged quantitatively, its value can only be deduced from observing 

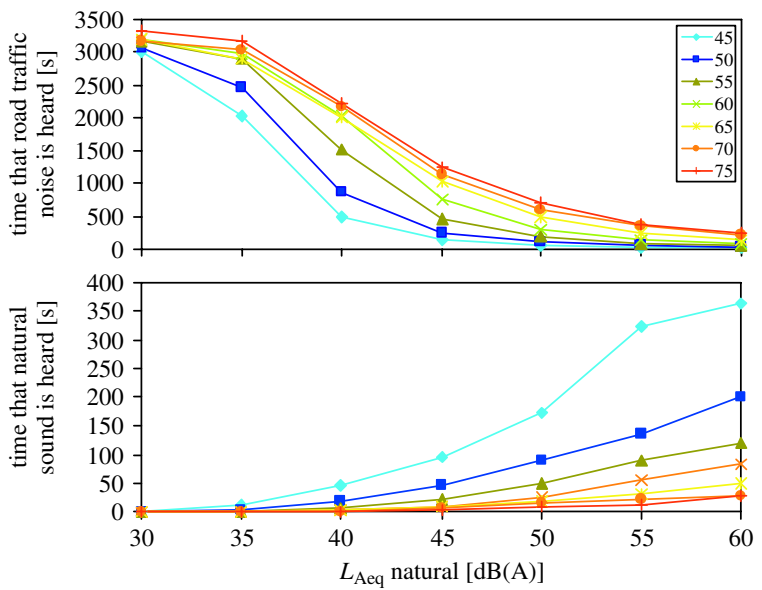

Fig. 4. Time that road traffic noise and natural sound is heard within one hour as a function of average natural ambient sound level for different average traffic noise levels; high top down attention for environmental sound.
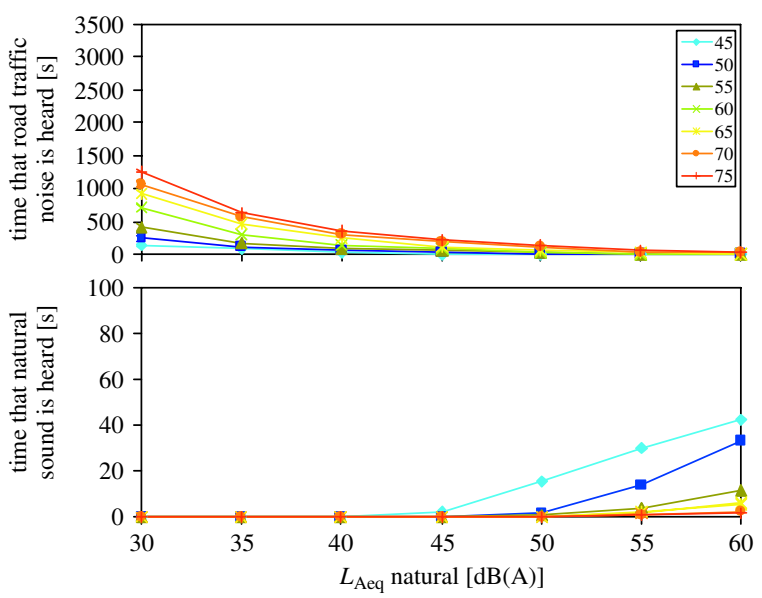

Fig. 5. Time that road traffic noise and natural sound is heard within one hour as a function of average natural ambient sound level for different average traffic noise levels; low top-down attention for environmental sound.

phenomenological effects. From the results in Fig. 4 it could be assumed that this level of attention corresponds to a trained environmental scientist observing the rural soundscape. The level of attention leading to Fig. 5 would correspond to that of the recreating visitor. With decreasing attention for environmental sound, the curves seem to shift over the $x$-axes. As bottom-up attention is ignored, the main reason for the observed trends is physiological masking or at least reduction of signal-to-noise ratio.

In a second numerical experiment, the level of bottom-up attention is increased to a level comparable to the difference between the two levels of top-down attention used above: $A_{\text {es }, d} \approx A_{\text {down }}^{(\text {high })}-A_{\text {down }}^{(\text {low }}$. In Fig. 6 and Fig. 7 results are shown for less volatile attention $(\beta=0.5)$ and more volatile attention $(\beta=1)$ respectively. On average, the time that environmental sounds are noticed increases with added
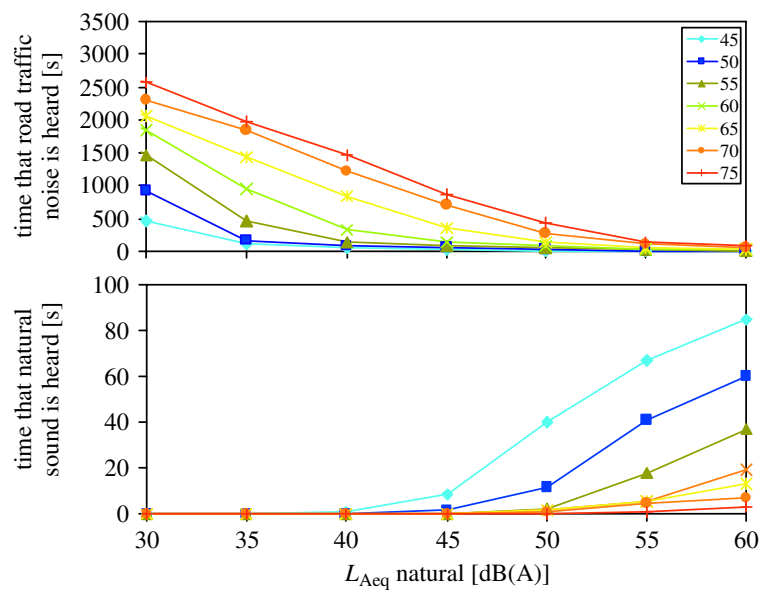

Fig. 6. Time that road traffic noise and natural sound is heard within one hour as a function of average natural ambient sound level for different average traffic noise levels; low top-down attention, $\beta=0.5$.
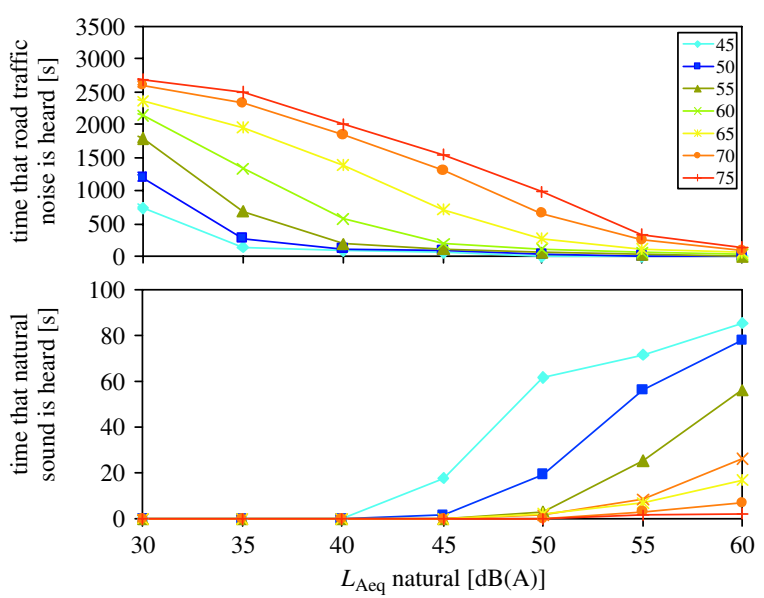

Fig. 7. Time that road traffic noise and natural sound is heard within one hour as a function of average natural ambient sound level for different average traffic noise levels; low top-down attention, $\beta=1$.

bottom-up attention, but the effect is different from the effect of increased top-down attention: the duration of noticing lower level traffic sound does not increase as much as the duration of hearing loud traffic sound. This can clearly be related to attracting more attention.

Differences between Figs. 6 and 7 are most pronounced in the region where neither the traffic sound nor the natural sound dominates. Indeed, a different type of environmental sound needs to be present for switching of attention between sounds to occur, and thus for volatile attention to show its effect.

To illustrate how bottom-up attention works, the hourly averaged level of bottom-up attention for road traffic noise is shown in Fig. 8 for different combinations of road traffic noise and background noise. The highest levels of bottomup attention are found for high road traffic noise levels 


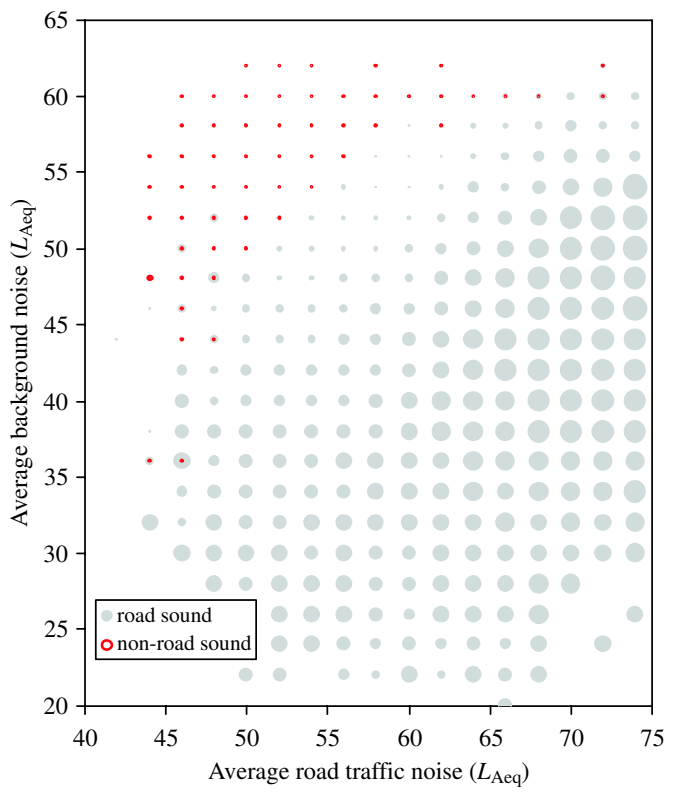

Fig. 8. Hourly averaged level of bottom-up attention (size of dots) for road traffic noise for different combinations of average road traffic noise level and background (all other sources) noise level; low top-down attention, $\beta=1$.

and moderate background levels. As the background level becomes higher, both physiological masking of road traffic sound and competition for attention kick in and the bottomup attention for road traffic noise reduces. At lower levels of background noise, a small decrease in attention is observed for all levels of road traffic noise. It is believed that this is due to habituation to road traffic noise.

\section{B. Combined exposure to rail and road sounds}

The second case study concerns the perception of the soundscape in an at-home context, in which individuals are exposed to a combination of road and railway traffic. The results of the numerical experiment discussed in this section will be compared to a recent nationwide retrospective questionnaire survey on annoyance conducted in Flanders, the northern part of Belgium [21]. About 7500 people completed an elaborated questionnaire containing about 100 questions, part of which handling noise annoyance caused by road and railway traffic during the last 12 months.

Using standard noise mapping software, the yearly average sound level $L_{\mathrm{den}}$ at the façade of the dwelling of all participants was calculated, taking into account all railways and main roads in Flanders. Fig. 9 shows the classical relationship between annoyance and road and railway traffic noise exposure. There is a clear difference in annoyance between road and railway traffic noise at the same average sound level (the so called railway bonus), a phenomenon that has been observed in several field studies in the past [22].

A synthetic population, resembling the population of the actual survey participants as close as possible, was constructed. Using the addresses of the dwellings of the

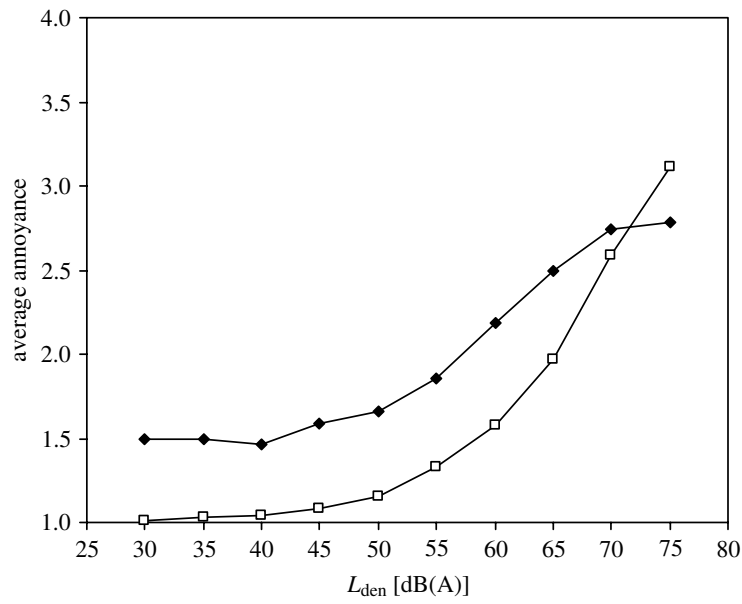

Fig. 9. Average annoyance of the survey population (5-point scale), caused by road $(\diamond)$ and railway traffic $(\square)$ noise as a function of average noise level at the façade.

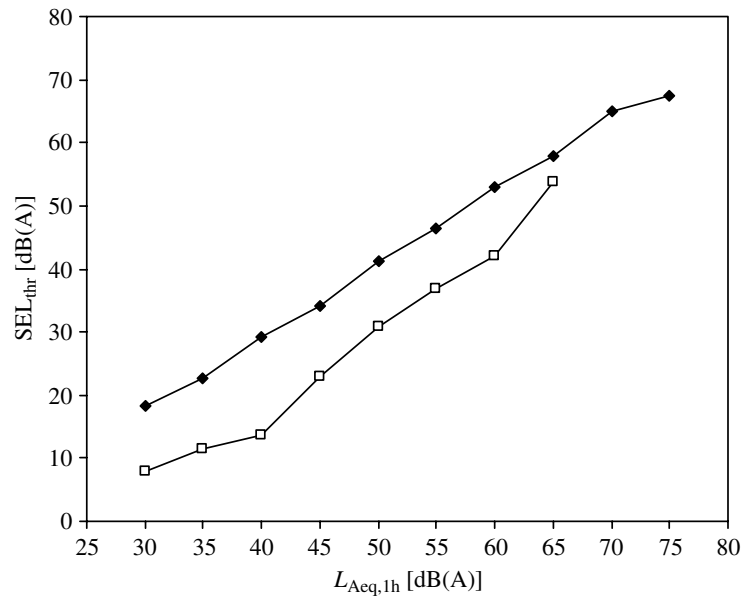

Fig. 10. Above-threshold exposure level of the sound noticed by the simulated individuals of the synthetic population, caused by road $(\downarrow)$ and railway traffic $(\square)$ noise exposure as a function of average noise level.

participants and available GIS data, the distance of each dwelling to the nearest main road and railway track was calculated, and the average hourly intensity of vehicles/trains during daytime on the road/track was determined. While at home, the modeled individuals may be engaged in various activities, and consequently may have a varying background attention to noise. Therefore, background attention was randomized between individuals. Dwelling acoustic insulation also differs between situations, but because of a lack of data on insulation of individual dwellings, this parameter was randomized between 0 (open window) and $30 \mathrm{~dB}(\mathrm{~A})$. The sound produced by the home activity (radio, television, cooking, playing children...) can not be modelled accurately, so a similar approach was used as the approach used to create the natural ambient sound in outdoor situation. 


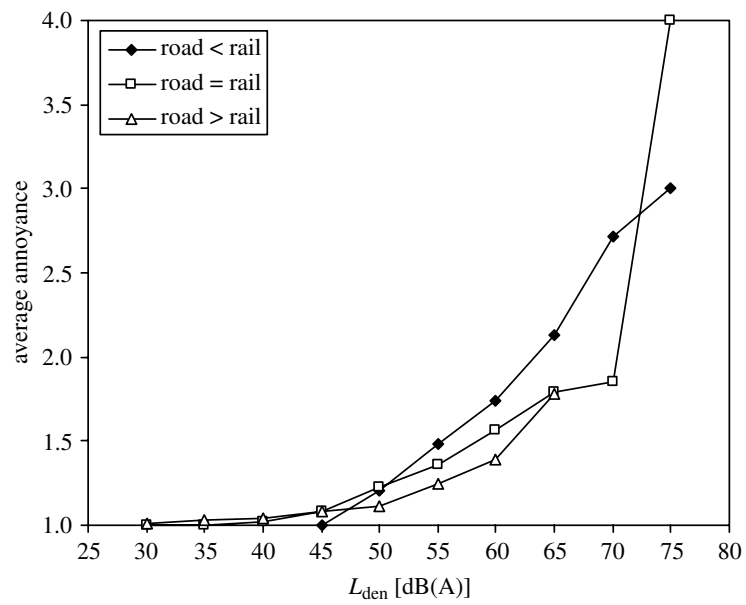

Fig. 11. Average annoyance of the survey population (5-point scale), caused by railway traffic noise exposure, split up for different relative levels of road traffic noise exposure.

Fig. 10 shows the above-threshold exposure level of the sound noticed by the simulated individuals of the synthetic population, as a function of road and railway traffic noise exposure. A difference between the curves for road and railway traffic noise at the same average sound level is found, similar to the survey results for annoyance.

Fig. 11 shows the survey exposure-effect relationship for railway noise annoyance, for exposure to railway noise higher, lower and approximately equal $( \pm 5 \mathrm{~dB})$ to road traffic noise exposure. It can be seen that, for equal average railway noise exposure level, railway noise annoyance is lower when road traffic noise exposure exceeds railway traffic noise exposure, at least for railway noise levels below $65 \mathrm{~dB}(\mathrm{~A})$. An even more pronounced trend is observed in the simulation results (Fig. 12).

How can these observations be explained in light of the discussed model? Consider the sound event caused by a train passage. With increasing level of the event, the probability that it is noticed and draws attention increases gradually. When train noise is considered on its own, habituation gradually decreases the probability of noticing, while focusing attention raises this probability. When road traffic noise is added to this picture, it will act as an additional background noise for noticing train passages. The reduced signal-to-noise ratio will decrease the overall probability of noticing. The attention mechanism complicates the picture. More detailed analyses shows that the crossing of curves at high exposure levels is accompanied by increased bottom-up attention for the (loud) train sounds. However, this mechanism is not reciprocal: train events, because of their relatively low rate of occurrence, are expected to have less influence on noticing road traffic noise.

\section{CONCLUSION}

In this paper it was shown how a rather simplified computer model for human auditory perception can be used to

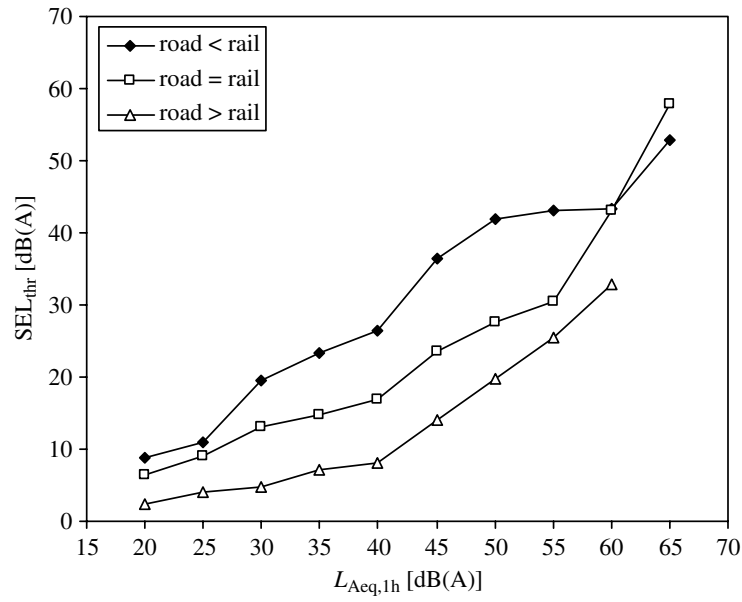

Fig. 12. Above-threshold exposure level of the sound noticed by the simulated individuals of the synthetic population, caused by railway traffic noise exposure, split up for different relative levels of road traffic noise exposure.

gain insight in the mechanisms underlying observed trends in the effect of environmental noise on people. From a modeling point of view, the influence of including bottomup attention leads to less abrupt transitions in combined exposure situations than a model solely based on perceptual masking would. The application to perception of open area soundscapes shows expected results for the influence of natural ambient sound levels. The application in an at-home environment leads to better understanding differences in annoyance caused by rail and road traffic and in particular gives insight into the combined effect of road and rail noise on people. For the latter, qualitative agreement with the results of a large survey was found.

\section{REFERENCES}

[1] J. B. Fritz, M. Elhilali, S. V. David, and S. A. Shamma, "Auditory attention - focusing the searchlight on sound," Current Opinion in Neurobiology, vol. 17, no. 4, pp. 437-455, 2007.

[2] B. Sagi, S. C. Nemat-Nasser, R. Kerr, R. Hayek, C. Downing, and R. Hecht-Nielsen, "A biologically motivated solution to the cocktail party problem," Neural Computation, vol. 13, no. 7, pp. 1575-1602, 2001.

[3] S. N. Wrigley and G. J. Brown, "A computational model of auditory selective attention," IEEE Transactions on Neural Networks, vol. 15, no. 5, pp. 1151-1163, 2004.

[4] B. Gygi and V. Shafiro, "General functions and specific applications of environmental sound research," Frontiers in Bioscience, vol. 12, pp. 3152-3166, 2007.

[5] L. Itti and C. Koch, "Computational modelling of visual attention," Nature Reviews Neuroscience, vol. 2, no. 3, pp. 194-203, 2001.

[6] R. M. Schafer, The soundscape: Our Sonic Environment and the Tuning of the World. Rochester, Vermont, USA: Destiny Books, 1994.

[7] D. Dubois, C. Guastavino, and M. Raimbault, "A cognitive approach to urban soundscapes: Using verbal data to access everyday life auditory categories," Acta Acustica united with Acustica, vol. 92, no. 6, pp. 865-874, 2006.

[8] B. De Coensel and D. Botteldooren, "The quiet rural soundscape and how to characterize it," Acta Acustica united with Acustica, vol. 92, no. 6, pp. 887-897, 2006. 
[9] B. Berglund and M. E. Nilsson, "On a tool for measuring soundscape quality in urban residential areas," Acta Acustica united with Acustica, vol. 92 , no. 6 , pp. 938-944, 2006.

[10] H. M. E. Miedema and H. Vos, "Noise sensitivity and reactions to noise and other environmental conditions," Journal of the Acoustical Society of America, vol. 113, no. 3, pp. 1492-1504, 2003.

[11] R. F. S. Job, J. Hatfield, A. J. Hede, P. Peploe, N. L. Carter, R. Taylor, and S. Morrell, "The role of attitudes and sensitivity in reactions to changing noise," in Proceedings of The 19th International Congress on Acoustics (ICA), Madrid, Spain, Sep. 2007.

[12] I. H. Flindell and P. J. M. Stillen, "Non-acoustical factors in environmental noise," Noise and Health, vol. 1, no. 3, pp. 11-16, 1999.

[13] B. De Coensel, T. De Muer, I. Yperman, and D. Botteldooren, "The influence of traffic flow dynamics on urban soundscapes," Applied Acoustics, vol. 66, no. 2, pp. 175-194, 2005.

[14] B. De Coensel, D. Botteldooren, and T. De Muer, " $1 / f$ noise in rural and urban soundscapes," Acta Acustica united with Acustica, vol. 89, no. 2, pp. 287-295, 2003.

[15] A. S. Bregman, Auditory Scene Analysis: The Perceptual Organization of Sound. Cambridge, Massachusetts, USA: The MIT Press, 1994.
[16] B. De Coensel, T. De Muer, and D. Botteldooren, "An agent based modeling approach to explain the perception of environmental stressors," in Proceedings of the 2007 International Conference on Artificial Intelligence (ICAI 2007), Las Vegas, Nevada, USA, Jun. 2007.

[17] R. Hecht-Nielsen, "Cogent confabulation," Neural Networks, vol. 18, no. 2, pp. 111-115, 2005.

[18] L. I. Perlovsky, "Toward physics of the mind: Concepts, emotions, consciousness, and symbols," Physics of Life Reviews, vol. 3, no. 1, pp. 23-55, 2006.

[19] H. Shi and Y. Yang, "A computational model of visual attention based on saliency maps," Applied Mathematics and Computation, vol. 188, no. 2, pp. 1671-1677, 2007.

[20] C. Kayser, C. Petkov, M. Lippert, and N. K. Logothetis, "Mechanisms for allocating auditory attention: An auditory saliency map," Current Biology, vol. 15, no. 21, pp. 1943-1947, 2005.

[21] "Schriftelijk leefomgevingsonderzoek $\mathrm{SLO}_{0} \& \mathrm{SLO}_{1}$ (in Dutch)," Environment, Nature and Energy Department (LNE), Flemish Government, Brussels, Belgium, Final report, Aug. 2004.

[22] H. M. E. Miedema and H. Vos, "Exposure-response relationships for transportation noise," Journal of the Acoustical Society of America, vol. 104 , no. 6 , pp. 3432-3445, 1998 


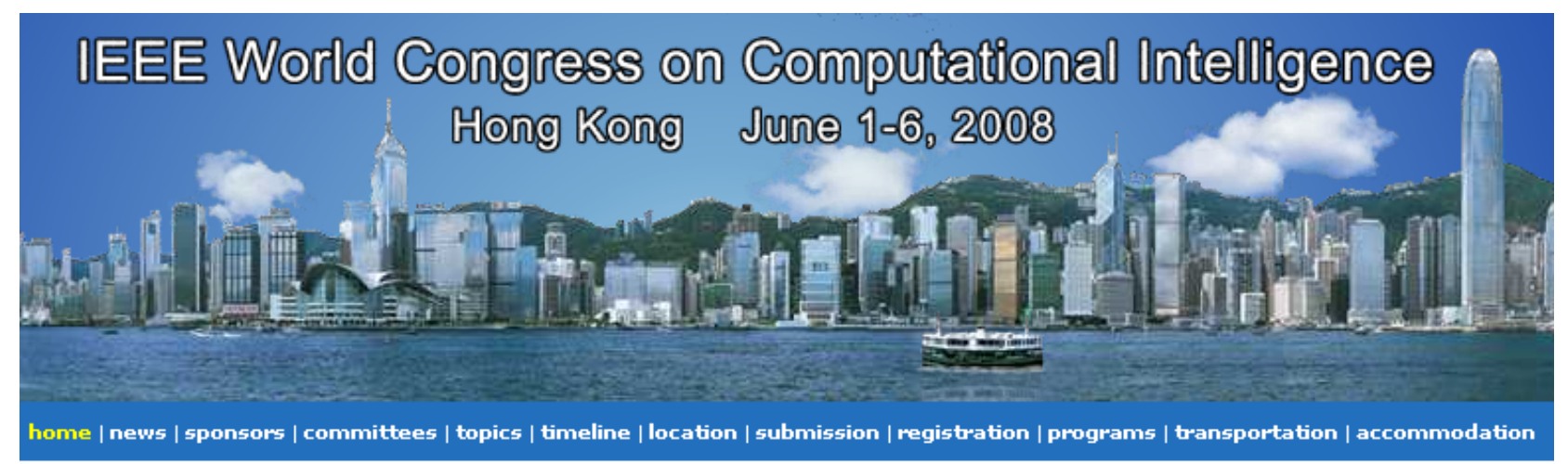

Messages from General Chair

On behalf of the Organizing Committee, I would like to thank you to participate in the 2008 IEEE World Congress on Computational Intelligence $(\mathrm{WCCl} 2008$ ). WCCl 2008 marks the fifth milestone in this series with a glorious history from $\mathrm{WCCl} 1994$ in Orlando, WCCl 1998 in Anchorage, $\mathrm{WCCl} 2002$ in Honolulu, to $\mathrm{WCCl} 2006$ in Vancouver. Sponsored by the IEEE Computational Intelligence Society and cosponsored by the International Neural Network Society, Evolutionary Programming Society, and the Institution of Engineering and Technology, $\mathrm{MCCl} 2008$ is the joint event of 2008 International Joint Conference on Neural Networks (IJCNN 2008), 2008 IEEE International Conference on Fuzy Systems (FUZZ-IEEE 2008), and 2008 IEEE Congress on Evolutionary Computation (CEC 2008). WCCl 2008 features the state-of-the-art plenary and invited speeches, parallel regular sessions, focused special sessions, interactive poster sessions, moderated panel discussions, informative pre-congress tutorials, complementary post-congress workshops, and entertaining social functions with following highlights:

- Five plenary speeches by world authorities and award recipients.

- Fifteen invited talks by renowned experts.

- Nine pre-congress tutorials by leading researchers in the fields with much reduced registration fees for widespread educational purposes.

- 2518 submissions from authors of over 70 countries across all inhabitable continents.

- Seventy nine advanced special sessions organized by domain specialists on focused topics.

- Eleven panels on a variety of topics to allow in-depth discussions and close interactions.

- Ten competitions in four categories on games, navigation, data mining, and optimization.

- Three post-congress workshops in Hong Kong and Macao for additional opportunities.

WCCl 2008 aims to provide a stimulating forum for thousands of scientists, engineers, educators, and students from all over the world to disseminate the latest research findings and exchange information on emerging areas of research in the fields. WCCl 2008 also creates a pleasant environment for you to meet your old friends for refreshing your memories and make new friends for expanding your network connections with possible future collaborations. Social events include a welcome reception, a women-in-computational-intelligence reception, an award banquet, a student and young graduate reception, and a farewell party. During the six days, we achieved a lot intellectually via intensive interactions and information exchanges. 